\title{
Corrigendum: Endothelin-1 supports clonal derivation and expansion of cardiovascular progenitors derived from human embryonic stem cells
}

Boon-Seng Soh, Shi-Yan Ng, Hao Wu, Kristina Buac, Joo-Hye C. Park, Xiaojun Lian, Jiejia Xu, Kylie S. Foo, Ulrika Felldin, Xiaobing He, Massimo Nichane, Henry Yang, Lie Bu, Ronald A. Li, Bing Lim \& Kenneth R. Chien

Nature Communications 7:10774 doi: 10.1038/ncomms10774 (2016); Published 8 Mar 2016; Updated 19 Jul 2016

The affiliation details for Boon-Seng Soh, Lei Bu and Ronald A. Li are incorrect in this Article. The correct addresses of these authors are listed below:

Boon-Seng Soh

Cardiovascular Research Center, Massachusetts General Hospital, 185 Cambridge Street, Boston, Massachusetts 02114, USA; Department of Stem Cell and Regenerative Biology, Harvard University, 7 Divinity Avenue, Cambridge, Massachusetts 02138, USA; Li Dak-Sum Research Centre-HKU-Karolinska Institutet Collaboration on Regenerative Medicine, University of Hong Kong, Hong Kong, China and Department of Cell and Molecular Biology and Medicine, Karolinska Institute, Stockholm S-171 77, Sweden.

Lei $\mathrm{Bu}$

Cardiovascular Research Center, Massachusetts General Hospital, 185 Cambridge Street, Boston, Massachusetts 02114, USA; Department of Stem Cell and Regenerative Biology, Harvard University, 7 Divinity Avenue, Cambridge, Massachusetts 02138, USA and Li Dak-Sum Research Centre-HKU-Karolinska Institutet Collaboration on Regenerative Medicine, University of Hong Kong, Hong Kong, China.

Ronald A. Li

Li Dak-Sum Research Centre-HKU-Karolinska Institutet Collaboration on Regenerative Medicine, University of Hong Kong, Hong Kong, China.

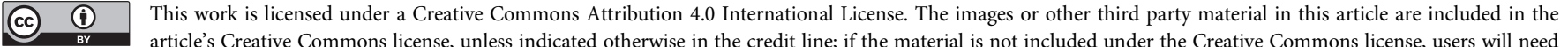
to obtain permission from the license holder to reproduce the material. To view a copy of this license, visit http://creativecommons.org/licenses/by/4.0/ 